S. Cole, J. Beerten and R. Belmans, "Generalized dynamic VSC MTDC model for power system stability studies," IEEE Trans. Power Syst., vol. 25, no. 3, pp. 1655-1662, Aug. 2010.

Digital Object Identifier: 10.1109/TPWRS.2010.2040846

URL:

http://ieeexplore.ieee.org/xpl/articleDetails.jsp?tp=\&arnumber=5411929

(C) 2010 IEEE. Personal use of this material is permitted. Permission from IEEE must be obtained for all other users, including reprinting/ republishing this material for advertising or promotional purposes, creating new collective works for resale or redistribution to servers or lists, or reuse of any copyrighted components of this work in other works. 


\title{
Generalized Dynamic VSC MTDC Model for Power System Stability Studies
}

\author{
Stijn Cole, Student Member, IEEE, Jef Beerten, Student Member, IEEE, Ronnie Belmans, Fellow, IEEE
}

\begin{abstract}
In this paper, a new general Voltage Source Converter High Voltage Direct Current (VSC MTDC) model is derived mathematically. The full system model consists of the converter and its controllers, DC circuit equations, and coupling equations. The main contribution of the new model is its valid for every possible topology of the DC circuit. Practical implementation of the model in power system stability software is discussed in detail. The generalized DC equations can all be expressed in terms of matrices that are byproducts of the construction of the DC bus admittance matrix. Initialization, switching actions resulting in different topologies and simulation of the loss of DC lines amount to a simple calculation or recalculation of the DC bus admittance matrix. The model is implemented in Matlab. Examples on a two- and six-terminal system show that the new model is indeed capable of accurately simulating VSC MTDC systems with arbitrary topology.
\end{abstract}

Index Terms-HVDC converters, HVDC transmission, Power system modelling, power system simulation

\section{INTRODUCTION}

W ITH an ever increasing number of installations, VSC HVDC systems become more and more important in the power system world. One advantage of VSC HVDC compared to conventional Current Source Converter (CSC) HVDC is that the extension to Multiterminal DC (MTDC) systems is relatively easy. Although up till now, there are no VSC MTDC systems installed or even in concrete planning phase, quite a number of publications are devoted to the subject of VSC MTDC. They deal with various aspects of MTDC operation such as location of DC faults and protection [1], [2], control strategy [3], harmonics [4], and applications in wind farms [5], [6]. Also, numerous papers exist on the modelling of two-terminal VSC HVDC systems [7], [8], [9], [10] and some on the modelling of multi-terminal CSC HVDC systems and their implementation in stability programs, e.g. [11] and [12]. As for basic modelling of VSC MTDC systems in power system stability programs, literature is limited to non-existant. The multi-terminal models that are used in the aforementioned papers on MTDC have a fixed topology. It is not explained how to extend the models to other topologies. In case of CSC HVDC, this would be understandable because topologies are limited to a few simple configurations anyhow, due to the inherent difficulties associated with extending CSCs

S. Cole is with the Department of Electrical Engineering, K.U.Leuven, Leuven, 3001 Belgium e-mail: stijn.cole@esat.kuleuven.be.

J. Beerten is with the Department of Electrical Engineering, K.U.Leuven, Leuven, 3001 Belgium. He is funded by a research grant from the Research Foundation Flanders (FWO) e-mail: jef.beerten@esat.kuleuven.be.

R. Belmans is with the Department of Electrical Engineering, K.U.Leuven, Leuven, 3001 Belgium, and chairman of the board of directors of Elia, Brussels, Belgium e-mail: ronnie.belmans@esat.kuleuven.be. to multi-terminal systems. Furthermore, there already exist two multi-terminal CSC HVDC systems. Research is mostly limited to the particular topologies of those existing systems. For VSCs the situation is quite different. Firstly, as we already mentioned, they can be easily extended to multi-terminal systems, possibly with a large number of converters. Secondly, it is hard to predict which topology future MTDC systems will have. While it can be conjectured that the first actual VSC MTDC system will have a simple topology with a limited number of converters, quite a wide variety of "supergrid" topologies are conceivable.

This paper aims to redress this lacuna by proposing a general dynamic model of VSC MTDC, valid for every conceivable topology of the DC circuit. The model allows adding or removing converters and lines without changing the model itself. The model is susceptible of integration with large step size, so that it can be used for the simulation of large scale AC/DC systems. A converter model including controls is first derived in section II. In section III, the general DC circuit equations are derived. It is explained in section IV how to combine the converter and DC circuit equations to represent any VSC MTDC system. In section V, practical implementation of the general MTDC equations in transient stability programs is discussed. The paper is concluded with simulations that show the generality of the model.

\section{CONVERTER Modelling}

VSC HVDC converters are connected to the system through a phase reactor, represented here by an impedance $Z_{p r}=$ $R_{p r}+j X_{p r}$, that can also include the effect of the transformer (Fig. 1). The basic equation of this circuit,

$$
u_{c}-u_{s}=L_{p r} \frac{d i_{p r}}{d t}+R_{p r} i_{p r}
$$

can be transformed to a power system synchronized rotating $d q$ reference frame:

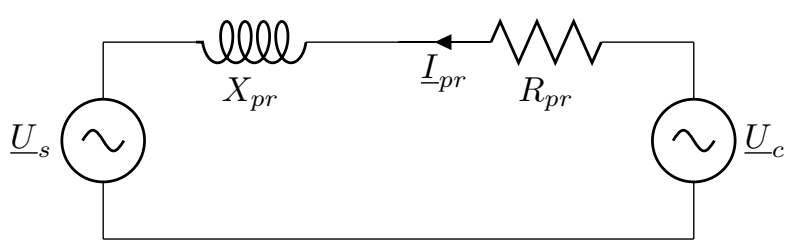

Fig. 1. One-line diagram of the AC Circuit. Subscript $\mathrm{s}$ for system side, subscript $\mathrm{c}$ for converter side 
$\frac{d\left(i_{p r}^{d} e^{j \omega t}\right)}{d t}=-\frac{R_{p r}}{L_{p r}} i_{p r}^{d} e^{j \omega t}+\omega i_{p r}^{q} e^{j \omega t}+\frac{1}{L_{p r}}\left(u_{c}^{d}-u_{s}^{d}\right) e^{j \omega t}$

$\frac{d\left(i_{p r}^{q} e^{j \omega t}\right)}{d t}-\frac{R_{p r}}{L_{p r}} i_{p r}^{q} e^{j \omega t}-\omega i_{p r}^{d} e^{j \omega t}+\frac{1}{L_{p r}}\left(u_{c}^{q}-u_{s}^{q}\right) e^{j \omega t}$

The angle $\omega t$ is provided by the phase-locked loop (PLL), arbitrarily assumed here to align system voltage with the qaxis. Dividing by $e^{j \omega t}$, the dynamic equations of the AC circuit in the $d q$-frame are finally obtained:

$$
\begin{aligned}
& \frac{d i_{p r}^{d}}{d t}=-\frac{R_{p r}}{L_{p r}} i_{p r}^{d}+\omega i_{p r}^{q}+\frac{1}{L_{p r}}\left(u_{c}^{d}-u_{s}^{d}\right), \\
& \frac{d i_{p r}^{q}}{d t}=-\frac{R_{p r}}{L_{p r}} i_{p r}^{q}-\omega i_{p r}^{d}+\frac{1}{L_{p r}}\left(u_{c}^{q}-u_{s}^{q}\right) .
\end{aligned}
$$

Most VSC converters have a cascaded control structure, comprising inner current control loop and outer controllers [13]. The controllers themselves are usually PI controllers, "the de facto industry standard for current control", that guarantee high performance ( [14]), and also used in e.g. [15]. In this paper, the emphasis is not placed on the control scheme. Therefore, a standard control scheme, consisting of cascaded PI controllers, is implemented here.

\section{A. Current Controllers}

The converter equations (3a) and (3b), obtained in the previous section can be controlled by two parallel control loops, using PI controllers. The cross-coupling terms,

$$
\begin{gathered}
\Delta u_{c}^{d}=\omega L_{p r} i_{p r}^{q} \\
\Delta u_{c}^{q}=-\omega L_{p r} i_{p r}^{d},
\end{gathered}
$$

compensate for the cross coupling between the two control loops, introduced in (3a), (3b) by the transformation to a rotating reference frame. The reference voltage in $\mathrm{d}$ and $\mathrm{q}$ components are then expressed as:

$$
\begin{aligned}
& u_{c^{r e f}}^{d}=u_{s}^{d}+\Delta u_{c}^{d}+\left(K_{p 1}+\frac{K_{i 1}}{s}\right)\left(i_{p r^{r e f}}^{d}-i_{p r}^{d}\right) \\
& u_{c^{r e f}}^{q}=u_{s}^{q}+\Delta u_{c}^{q}+\left(K_{p 1}+\frac{K_{i 1}}{s}\right)\left(i_{p r^{r e f}}^{q}-i_{p r}^{q}\right) .
\end{aligned}
$$

After introducing two state variables, $M_{d}$ and $M_{q}$, such that

$$
\begin{aligned}
& \frac{d M_{d}}{d t}=K_{i 1}\left(i_{p r^{r e f}}^{d}-i_{p r}^{d}\right) \\
& \frac{d M_{q}}{d t}=K_{i 1}\left(i_{p r \text { ref }}^{q}-i_{p r}^{q}\right),
\end{aligned}
$$

the current controller equations become

$$
\begin{aligned}
& u_{c^{r e f}}^{d}=u_{s}^{d}+\Delta u_{c}^{d}+K_{p 1}\left(i_{p r^{r e f}}^{d}-i_{p r}^{d}\right)+M_{d}, \\
& u_{c^{r e f}}^{q}=u_{s}^{q}+\Delta u_{c}^{q}+K_{p 1}\left(i_{p r^{r e f}}^{q}-i_{p r}^{q}\right)+M_{q} .
\end{aligned}
$$

The actual value of the voltage lags the reference due to the time-lag of the converter's power electronics. The relation between the actual value and the reference value can be represented by a time delay with time constant $T_{\sigma}$ [14]:

$$
\frac{U_{c}^{d q}}{U_{c^{r e f}}^{d q}}=\frac{1}{T_{\sigma} s+1}
$$

or in the time-domain:

$$
\begin{aligned}
\frac{d u_{c}^{d}}{d t} & =\frac{1}{T_{\sigma}}\left(u_{c^{r e f}}^{d}-u_{c}^{d}\right), \\
\frac{d u_{c}^{q}}{d t} & =\frac{1}{T_{\sigma}}\left(u_{c^{r e f}}^{q}-u_{c}^{q}\right) .
\end{aligned}
$$

Combining the time-lag equations (10a), (10b) and the equations of the current controllers $(8 \mathrm{a}),(8 \mathrm{~b})$ yields:

$$
\begin{aligned}
& \frac{d u_{c}^{d}}{d t}=-\frac{K_{p 1}}{T_{\sigma}} i_{p r}^{d}-\omega \frac{L_{p r}}{T_{\sigma}} i_{p r}^{q}-\frac{1}{T_{\sigma}} u_{c}^{d}+ \\
& \frac{1}{T_{\sigma}} M_{d}+\frac{K_{p 1}}{T_{\sigma}} i_{p r e f}^{d}+\frac{1}{T_{\sigma}} u_{s}^{d}, \\
& \frac{d u_{c}^{q}}{d t}=-\frac{K_{p 1}}{T_{\sigma}} i_{p r}^{q}+\omega \frac{L_{p r}}{T_{\sigma}} i_{p r}^{d}-\frac{1}{T_{\sigma}} u_{c}^{q}+ \\
& \frac{1}{T_{\sigma}} M_{q}+\frac{K_{p 1}}{T_{\sigma}} i_{p r e f}^{q}+\frac{1}{T_{\sigma}} u_{s}^{q} .
\end{aligned}
$$

\section{B. Outer Controllers}

1) Reactive Power Control and Voltage Control: In a VSC HVDC system, every converter can independently control its reactive power injection in the power system. When the system voltage is aligned with the q-axis, the reactive power $Q$ can be calculated as follows:

$$
Q=u_{s}^{q} i_{p r}^{d}
$$

The d-axis current setpoint, $i_{p r e f}^{d}$, is thus calculated from the reactive power setpoint $Q_{r e f}$. A combination of an open loop and a PI controller is used to drive reactive power to its desired value, leading to the equation [16]:

$$
i_{p r \text { ref }}^{d}=\frac{Q_{r e f}}{u_{s}^{q}}+\left(K_{p 2}+\frac{K_{i 2}}{s}\right)\left(Q_{r e f}-Q\right) .
$$

We finally introduce state variable $N^{d}$, resulting in an additional differential equation for the PI controller,

$$
\frac{d N^{d}}{d t}=K_{i 2}\left(Q_{r e f}-u_{s}^{q} i_{p r}^{d}\right),
$$

such that substitution of (12) and (14) in (13) leads to an expression for the $\mathrm{d}$-axis current reference:

$$
i_{p r^{r e f}}^{d}=\frac{Q_{r e f}}{u_{s}^{q}}+N^{d}+K_{p 2}\left(Q_{r e f}-u_{s}^{q} i_{p r}^{d}\right),
$$

which can thereafter be substituted in (11a).

Instead of controlling reactive power, the AC system voltage can be controlled. The d-axis current reference can be calculated in a similar manner:

$$
i_{p r^{r e f}}^{d}=\left(K_{p 3}+\frac{K_{i 3}}{s}\right)\left(U_{s^{r e f}}-U_{s}\right),
$$


with

$$
U_{s}=\sqrt{\left(u_{s}^{q}\right)^{2}+\left(u_{s}^{d}\right)^{2}}=u_{s}^{q} .
$$

After we introduce state variable $N^{d}$, the following equations can be written down:

$$
\begin{gathered}
\frac{d N^{d}}{d t}=K_{i 3}\left(U_{r e f}-u_{s}^{q}\right), \\
i_{p r^{r e f}}^{d}=N^{d}+K_{p 3}\left(U_{r e f}-u_{s}^{q}\right) .
\end{gathered}
$$

Equation (19) can be substituted in (11a).

2) Active Power Control: In a two-terminal VSC HVDC system, one converter sets the active power setpoint. The variable $i_{p r r e f}^{q}$ is calculated from this active power setpoint. The other converter controls the DC voltage. We call it the 'slack' converter, because it compensates the losses in the DC network and thus has a similar function as a slack node in an AC grid. In general, an $n$ converter VSC HVDC system has $n-1$ converters controlling active power, and one controlling DC voltage. One must therefore differentiate between the slack converter and the other converters for the calculation of the $\mathrm{q}$-axis current setpoint. As only $n-1$ converters can set an active power setpoint, $n-1 \mathrm{q}$-axis reference currents can be calculated from the active power setpoint. As we will explain in section IV, the remaining q-axis reference current can be obtained from the DC voltage control equation of the slack converter. Again using a combination of an open loop and a PI controller, the q-axis reference current can be expressed as:

$$
i_{p r^{r e f}}^{q}=\frac{P_{r e f}}{u_{s}^{q}}+\left(K_{p 4}+\frac{K_{i 4}}{s}\right)\left(P_{r e f}-P\right),
$$

with

$$
P=u_{s}^{q} i_{p r}^{q} .
$$

Introducing a new state variable $N^{q}$ leads to an extra equation,

$$
\frac{d N^{q}}{d t}=K_{i 4}\left(P_{r e f}-u_{s}^{q} i_{p r}^{q}\right) .
$$

After substitution of (22) and (21) in (20), the expression for the q-axis reference current to be substituted in (11b) becomes:

$$
i_{p r r e f}^{q}=\frac{P_{r e f}}{u_{s}^{q}}+N^{q}+K_{p 4}\left(P_{r e f}-u_{s}^{q} i_{p r}^{q}\right) .
$$

All the equations of the converters are now derived.

\section{DC CirCuit Modelling}

The DC circuit of a VSC HVDC system consists of a large capacitor at the converter station and a DC cable. Fig. 2 represents the DC side of a two-terminal VSC HVDC system with two cables of opposite voltage level. The basic equations are:

$$
\begin{aligned}
& C_{d c} \frac{d u_{d c_{1}}}{d t}=i_{d c_{1}}-i_{c c}, \\
& C_{d c} \frac{d u_{d c_{2}}}{d t}=i_{d c_{2}}+i_{c c},
\end{aligned}
$$

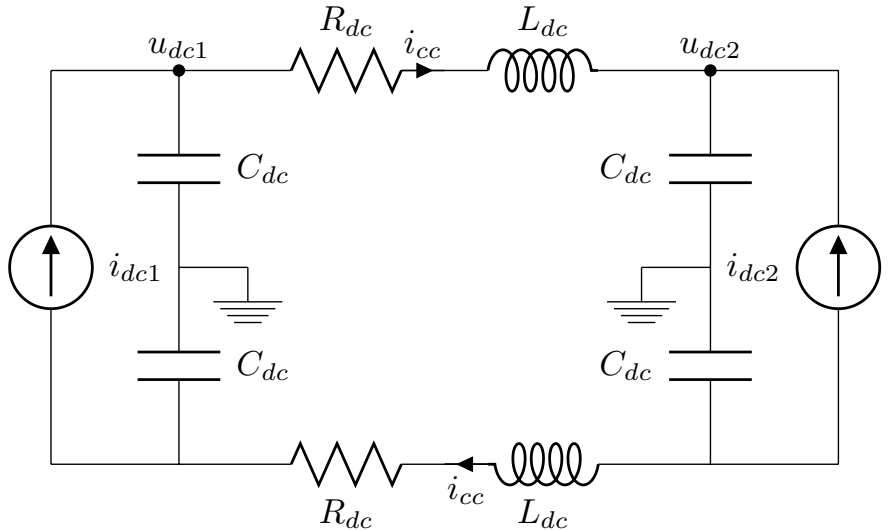

Fig. 2. DC Circuit of two-terminal VSC HVDC system

$$
L_{d c} \frac{d i_{c c}}{d t}=u_{d c_{1}}-u_{d c_{2}}-R_{d c} i_{c c} .
$$

A converter of a MTDC system can be connected to a number of other converters (Fig. 3). We fix the current directions in the DC lines such that the current from converter $i$ to converter $j$ is positive if $i$ is smaller than $j$. Converter 1 has thus only incoming currents, and converter $n$ only outgoing currents. For every other converter $i$, there are $n-1$ incoming, and $n-i$ outgoing currents. The generalized dynamic equations of any DC circuit can then be written as:

$$
\begin{aligned}
& C_{d c_{i}} \frac{d u_{d c_{i}}}{d t}=i_{d c_{i}}-\sum_{j=i+1}^{n} i_{c c_{i j}}, \quad i=1 \\
& C_{d c_{i}} \frac{d u_{d c_{i}}}{d t}=i_{d c_{i}}+\sum_{j=1}^{i-1} i_{c c_{j i}}-\sum_{j=i+1}^{n} i_{c c_{i j}}, \quad i=2, \ldots, n-1
\end{aligned}
$$

$C_{d c_{i}} \frac{d u_{d c_{i}}}{d t}=i_{d c_{i}}+\sum_{j=1}^{i-1} i_{c c_{j i}}, \quad i=n$

and

$$
L_{d c} \frac{d i_{c c_{i j}}}{d t}=u_{d c_{i}}-u_{d c_{j}}-R_{d c_{i j}} i_{c c_{i j}} \quad \forall j<n, \forall i<j .
$$

The DC voltage control equations have to be appended to the above equations. The slack converter controls the DC voltage. When a PI controller is used, the reference DC current of the slack converter can be expressed as:

$$
i_{d c_{n}^{r e f}}=\left(K_{p}+\frac{K_{i}}{s}\right) \cdot\left(u_{d c r e f}-u_{d c_{n}}\right) .
$$

For the control of the DC voltage, the dynamics of the DC circuit are approximated by a time constant $T_{d c}$, determined by the DC capacitors.

$$
\frac{i_{d c_{n}}}{i_{d c_{n}^{r e f}}}=\frac{1}{T_{d c} s+1} .
$$

Combining this equation with (29), and introducing

$$
\frac{d M_{d c}}{d t}=K_{i d c}\left(u_{d c r e f}-u_{d c_{n}}\right),
$$




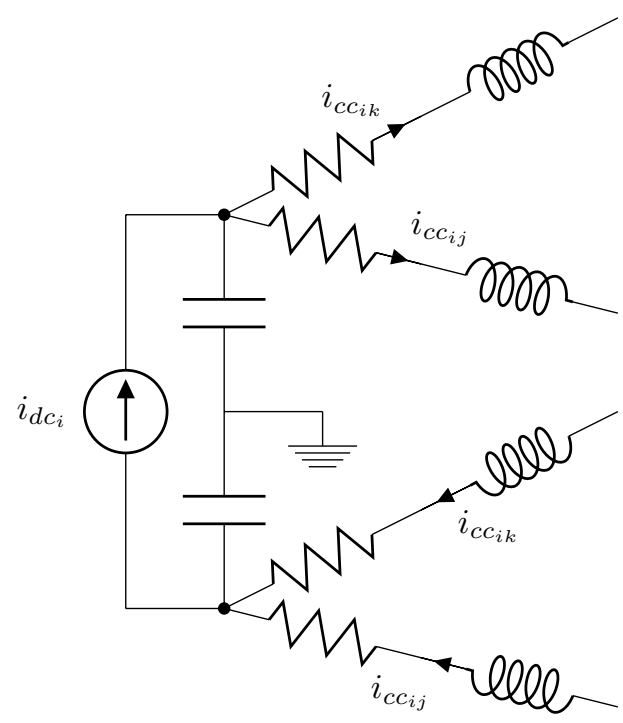

Fig. 3. Node in a MTDC Circuit

the following control equation is obtained:

$$
\frac{d i_{d c_{n}}}{d t}=\frac{1}{T_{d c}}\left(-i_{d c_{n}}+K_{p d c}\left(u_{d c r e f}-u_{d c_{n}}\right)+M_{d c}\right)
$$

\section{Full Model}

\section{A. Full Model Equations}

Finally, the AC and DC circuit equations have to be coupled. The coupling equations follow from the active power balance between $\mathrm{AC}$ and $\mathrm{DC}$ side of the converter. They allow to calculate the q-axis reference current of the slack converter, and the dc currents of the other converters:

$$
\begin{gathered}
i_{p r_{n}^{r e f}}^{q}=\frac{2 i_{d c_{n}} \cdot u_{d c_{n}}-i_{p r_{n}}^{d} \cdot u_{c_{n}}^{d}}{u_{c_{n}}^{q}}, \\
i_{d c_{i}}=\frac{u_{c_{i}}^{d} \cdot i_{p r_{i}}^{d}+u_{c_{i}}^{q} \cdot i_{p r_{i}}^{q}}{2 u_{d c_{i}}}, \quad \forall i \leq n-1 .
\end{gathered}
$$

The full model of a VSC MTDC system is obtained by combining the equations of the converters with their current and outer controllers, DC circuit equations, and the AC-DC coupling equations. A general $n$ convertor system is composed of one slack converter, defined by the equations in Table I, $n-1$ converters defined by the equations in Table II, and a DC circuit (Table III).

TABLE I

EQUATIONS SLACK CONVERTER

\begin{tabular}{c|c}
\hline Converter & $(3)$ \\
\hline I controller & $(11),(7)$ \\
\hline Q controller & $(14),(15)$ \\
or & or \\
U controller & $(18),(19)$ \\
\hline Coupling & $(33)$ \\
\hline
\end{tabular}

TABLE II

EQUATIONS OTHER CONVERTERS

\begin{tabular}{c|c}
\hline Converter & $(3)$ \\
\hline I controller & $(11),(7)$ \\
\hline Q controller & $(14),(15)$ \\
or & or \\
U controller & $(18),(19)$ \\
\hline P controller & $(22),(23$ \\
\hline Coupling & $(34)$ \\
\hline
\end{tabular}

TABLE III

DC CIRCUIT EQUATIONS

\begin{tabular}{c|c}
\hline DC circuit & $(27),(28)$ \\
\hline DC controller & $(31),(32)$ \\
\hline
\end{tabular}

\section{B. Validity of the Model}

The main application of this model is the dynamic simulation of AC systems connected to large scale, meshed VSC MTVDC systems with many converters, with emphasis on transient stability studies. Therefore, the level of detail of the HVDC system model is limited, which allows fairly large step sizes, necessary for the simulation of large scale AC/DC systems. The model can be used to study the influence of the VSC MTDC system on the AC system, and the dynamics of the DC circuit. However, it should be noted that, as stated in the introduction, the phasor modelling approach is used in this paper, as is customary in transient stability studies. As a result, only information about the fundamental frequency components can be obtained from this model; it is not the aim of the proposed model to study phenomena such as harmonic transfer ( [4]). When information about harmonics is needed, a detailed converter model should be used, usually implemented in an EMTP type program, and solved with very small step size.

In Fig. 4, the response to a step in DC voltage reference of a detailed electromagnetic model, including detailed representation of three-level Neutral Point Clamped (NPC) VSC converters with sinusoidal pulse width modulation, control systems and PLL, is shown [17].

In Fig. 5, a typical respons of a phasor model to a same step in DC voltage reference is shown. The high frequency information is absent, but the step size can be much larger.

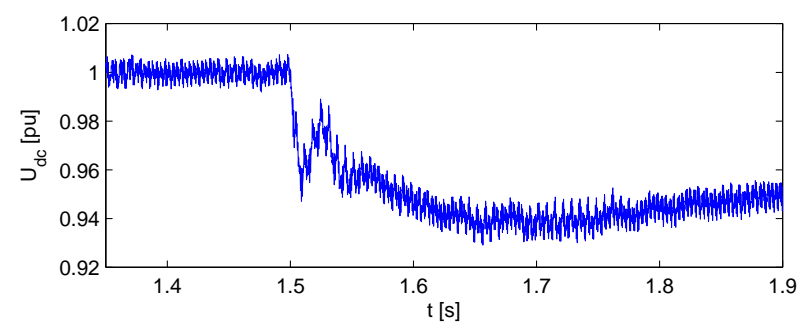

Fig. 4. DC voltage: detailed EMTP type model (step size $7.4 \mu s$ ). 


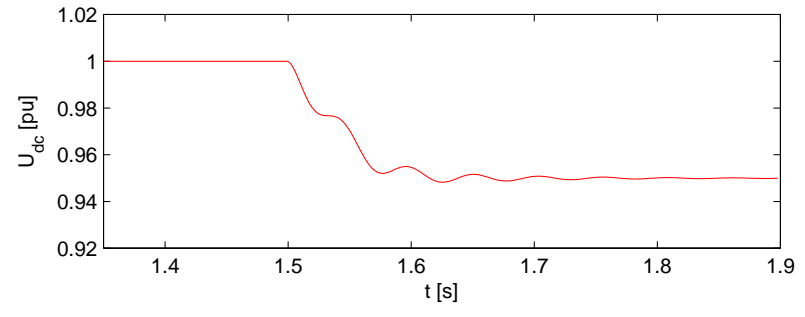

Fig. 5. DC voltage: phasor model (step size $1 \mathrm{~ms}$ ).

\section{Practical ImPLEMENTATION}

\section{A. Steady-State}

The steady-state DC network equations are

$$
\begin{gathered}
\mathrm{I}_{\mathrm{dc}}=\mathrm{Y}_{\mathrm{dc}} \mathrm{U}_{\mathrm{dc}}, \\
P_{d c_{i}}=2 U_{d c_{i}} I_{d c_{i}}, \quad \forall i \leq n .
\end{gathered}
$$

$\mathrm{Y}_{\mathrm{dc}}$ is the bus admittance matrix of the DC network, constructed using line resistances only.

The equations can be combined to form a system of nonlinear equations:

$$
0=\mathrm{Y}_{\mathrm{dc}} \mathrm{U}_{\mathrm{dc}}-\left[\frac{P_{d c_{i}}}{2 U_{d c_{i}}}\right] .
$$

As one DC bus controls DC voltage and the others active power, the problem has to be rearranged. To simplify notation, but without loss of generality, we assume that the last converter controls DC voltage. The vector of the unknowns is then

$$
\boldsymbol{X}=\left[\frac{\boldsymbol{X}_{\mathbf{1}}}{X_{2}}\right]=\left[\begin{array}{c}
U_{d c_{1}} \\
\vdots \\
\frac{U_{d c_{(n-1)}}}{P_{d c_{n}}}
\end{array}\right]
$$

The DC bus admittance matrix is partitioned as follows:

$$
\mathrm{Y}_{\mathrm{dc}}=\left[\begin{array}{c|c}
\mathrm{Y}_{11} & \boldsymbol{Y}_{\mathbf{1 2}} \\
\hline \mathbf{Y}_{\mathbf{2 1}} & y_{22}
\end{array}\right]
$$

The matrix $Y_{11}$ is of dimension $(n-1) \times(n-1), \boldsymbol{Y}_{12}$ is a column vector of length $n-1, \boldsymbol{Y}_{\mathbf{2 1}}$ a row vector of length $n-1$, and $y_{22}$ a scalar.

This leads to a system of equations that can be solved by a Newton method:

$$
\left\{\begin{array}{l}
0=Y_{11} \boldsymbol{X}_{\mathbf{1}}+\boldsymbol{Y}_{\mathbf{1 2}} \cdot u_{d c r e f}-P_{d c_{i}} /\left(2 X_{1 i}\right) \\
0=\boldsymbol{Y}_{\mathbf{2 1}} \boldsymbol{X}_{\mathbf{1}}+y_{22} \cdot u_{d c r e f}-X_{2} /\left(2 u_{d c r e f}\right)
\end{array}\right.
$$

Once the DC voltages are known, the currents between the DC buses can be obtained by premultiplying the DC bus voltage vector by $Y_{\text {dcf }}$ or $Y_{d c t}$. $Y_{\text {dcf }}$ is the matrix which, multiplied by the voltage vector, gives the currents leaving the buses (41). Analogously, $Y_{\text {dct }}$ relates bus voltages and currents entering the buses (42).

$$
\begin{aligned}
& \mathrm{I}_{\mathrm{ccf}}=\mathrm{Y}_{\mathrm{dcf}} \mathrm{U}_{\mathrm{dc}} \\
& \mathrm{I}_{\mathrm{cct}}=\mathrm{Y}_{\mathrm{dct}} \mathrm{U}_{\mathrm{dc}}
\end{aligned}
$$

$Y_{\text {dcf }}$ and $Y_{\text {dct }}$ are obtained as byproducts of the construction of the DC bus admittance matrix $Y_{d c}$ without extra computations.

\section{B. Dynamics}

Eq. (27) can be written as a matrix equation:

$$
\left[C_{d c_{i}} \frac{d U_{i}}{d t}\right]=\mathrm{I}_{\mathrm{dc}}+\mathrm{Cl}_{\mathrm{cc}}
$$

The matrix $C$ is the $n \times m$ incidence matrix of the directed graph describing the DC network. The incidence matrix of a directed graph is defined as a matrix with element $c_{i j}=-1$ if line $\mathrm{i}$ leaves node $\mathrm{j}, c_{i j}=1$ if line $\mathrm{i}$ enters node $\mathrm{j}$ and $c_{i j}=0$ otherwise. This matrix can be obtained as a byproduct of the construction of the DC bus admittance matrix $Y_{d c}$.

\section{Events}

The proposed formulation allows easy inclusion of events such as the loss of DC lines. The loss of a DC line can be simulated by removing the line from the power flow data and simply recalculating $Y_{d c}$, which also gives matrices $Y_{d c f}, Y_{d c t}$ and the network incidence matrix $C$. The dynamic equations (43) are automatically updated.

\section{Simulations}

\section{A. Two-terminal system}

The proposed model is implemented in Matlab. All simulations are performed using modified versions of Matpower [18] and MatDyn [19], [20]. MatDyn is an open-source program for dynamic simulation of power systems in Matlab. The ODEs are solved with a Modified Euler method with time step $1 \mathrm{~ms}$. Eq. (27) can be represented by a matrix equation, (43), that can be conveniently entered in a Matlab based toolbox such as MatDyn. If this equation were to be implemented in all its generality in a commercial grade power system software, it should be implemented at the source code level by the developer, as it would be very difficult or even impossible to use block-diagram based editors of commercial power system software.

We first consider a two-terminal VSC HVDC system installed in a 9-bus, 2-generator network (Fig. 12). The one-line diagram and data of this small power system are included in the appendix. A VSC HVDC link, described by the proposed model, is installed between buses two and three. At $t=0.2$, the DC voltage setpoint is changed. In Fig. 6 it can be seen that the new reference value is reached after a small transient. The transient propagates to the AC side: the VSC HVDC system follows the DC voltage step by charging the capacitors, and thus increasing the voltage. Hence, a change in DC voltage setpoint has an influence on active power exchange, as can be seen in Fig. 7. In Fig. 8, the influence on the system voltages is shown. At the inverter side, the transient is higher because this converter controls the DC voltage.

\section{B. Multi-terminal system}

A six-terminal VSC HVDC system is now simulated to test the proposed model. The sixth converter controls the DC voltage, the five other converters control active power. The DC circuit consists of seven lines, line 1-2, 1-6, 2-3, 3-4, 4-5, 4-6, and 5-6 (Fig. 9). For simplicity, the parameters of the all 


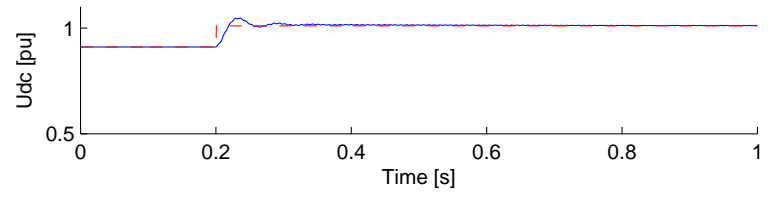

Fig. 6. DC voltage of the two-terminal system. Dotted lines are reference values and full lines real values.
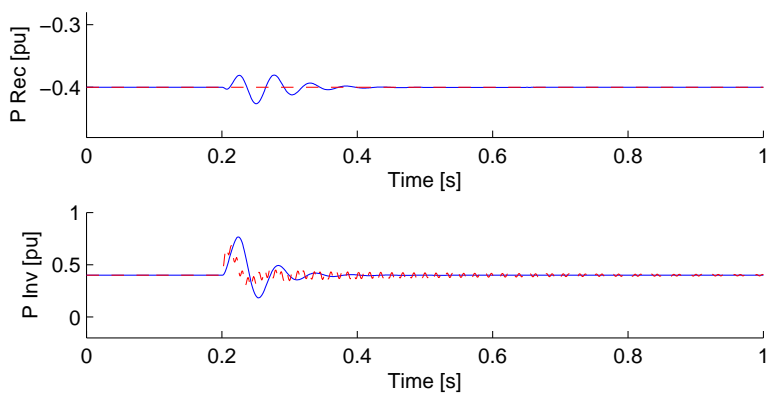

Fig. 7. Active power at rectifier and inverter of the two-terminal system. Dotted lines are reference values and full lines real values.

the DC lines are equal, and it is assumed that the converters are connected to a strong $\mathrm{AC}$ system, modelled by infinite nodes. However, the validity of the model is not dependent on these conditions. At $t=0.2$, the loss of line 5-6 is simulated. The current through the line drops to zero, and is distributed to the other lines (Fig. 10). Observing Fig. 9, it is expected that the loss of line 5-6, needs to be compensated for by a rise in current through lines 1-6 and 4-6. It can be verified from Fig. 10 that this is indeed what happens. In Fig. 11 the response of the DC voltage is shown. As a result of the changed flows, the DC voltages settle to new values after the transient. Only the voltage of the regulated converter, $U_{6}$, returns to its pre-fault value.

\section{CONCLUSION}

Simulations on a two-terminal ans six-terminal system have shown that the dynamic behaviour of VSC MTDC systems with arbitrary topology can be satisfactorily simulated by the generalized dynamic VSC MTDC model proposed in this paper. The basic equations of the converters and its controllers were first derived, whereby distinction had to be made between
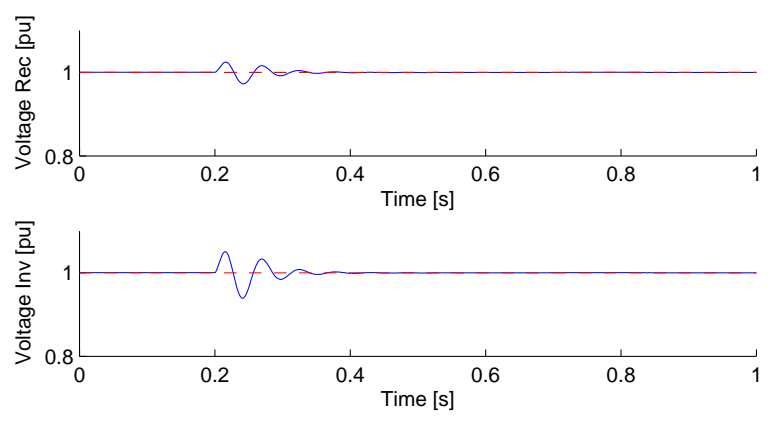

Fig. 8. System voltages at rectifier and inverter of the two-terminal system. Dotted lines are reference values and full lines real values.

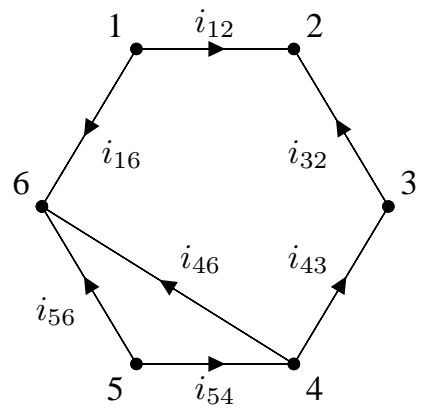

Fig. 9. Topology of six-terminal system

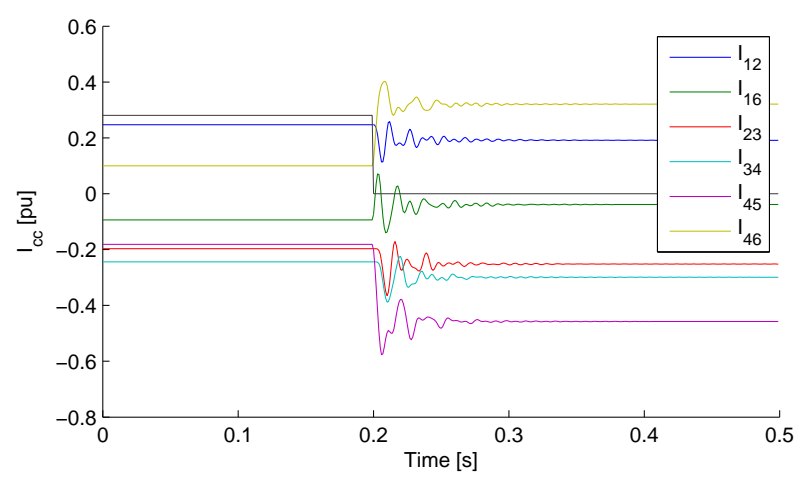

Fig. 10. DC currents of the six-terminal system.

the slack converter, which controls DC voltage, and the other converters, which control active power. Next, the equations of the DC system have been derived for the well-known twoterminal system, which were subsequently generalized. Lastly, the coupling equations between the $\mathrm{AC}$ and $\mathrm{DC}$ system were derived. By combining these equations, the complete system of equations is obtained. The main contribution of this paper is, that this system of equations is valid for every possible VSC HVDC multiterminal system, independent of its topology. This means that converters and lines can be added or removed during the simulation, without changing the model itself. The model can be integrated with large step size and is perfectly suited for the study of large AC/DC systems. Furthermore, we have explained how this model can be efficiently implemented in existing power system software, how the initial conditions

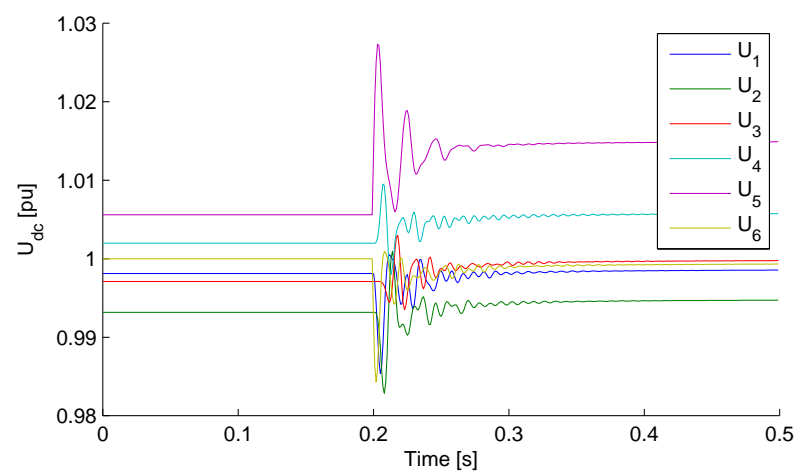

Fig. 11. DC Voltages of the six-terminal system. 
of the generalized DC system can be obtained and how faults can be taken into account. The range of validity and limitations of the model were discussed. It has been shown by numerical examples that VSC MTDC systems with different topologies can indeed been represented by the same system of equations. A change of DC voltage reference has been simulated for a two-terminal system and the loss of a DC line for a sixterminal system. The results have shown the model's good performance. It has been verified that the model is indeed valid for DC grids with arbitrary topologies.

\section{APPENDIX: POWER SyStem DATA}

The one-line diagram of the power system, used for the simulation of the two-terminal VSC HVDC system, is shown in Fig. 12. The power system data is given in the tables below.

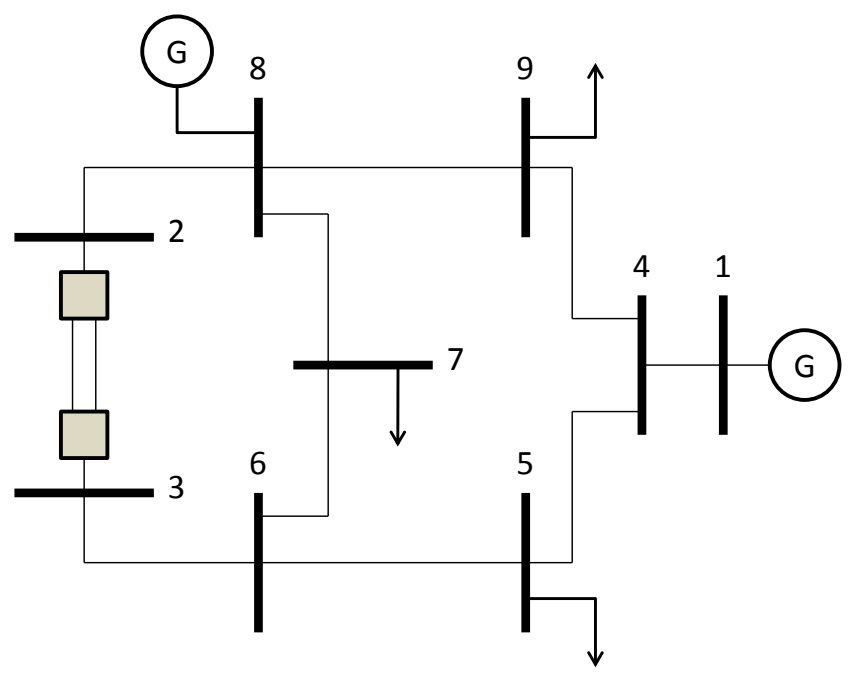

Fig. 12. Simulation two-terminal system: system layout.

Transmission line data

\begin{tabular}{c|c||c|c|c}
\hline From & To & R [pu] & X [pu] & B [pu] \\
\hline \hline 1 & 4 & 0 & 0.0576 & 0 \\
\hline 4 & 5 & 0.017 & 0.092 & 0.158 \\
\hline 5 & 6 & 0.039 & 0.17 & 0.358 \\
\hline 3 & 6 & 0 & 0.0586 & 0 \\
\hline 6 & 7 & 0.0119 & 0.1008 & 0.209 \\
\hline 7 & 8 & 0.0085 & 0.072 & 0.149 \\
\hline 8 & 2 & 0 & 0.0625 & 0 \\
\hline 8 & 9 & 0.032 & 0.161 & 0.306 \\
\hline 9 & 4 & 0.01 & 0.085 & 0.176 \\
\hline \multicolumn{5}{|c|}{ Load data } \\
\cline { 2 - 5 } & Bus & P [MW] & Q [MW] \\
\cline { 2 - 5 } & 5 & 90 & 30 \\
\cline { 2 - 5 } & 7 & 100 & 35 \\
\cline { 2 - 5 } & 9 & 125 & 50 \\
\cline { 2 - 5 }
\end{tabular}

Generator data

\begin{tabular}{c||c|c|c|c|c|c|c|}
\hline Bus & $\mathrm{H}$ & $\mathrm{D}$ & $x_{d}$ & $x_{q}$ & $x^{\prime}$ & $T_{d}^{\prime}$ & $T_{q}^{\prime}$ \\
\hline \hline 1 & 3.74 & 0.02 & 1 & 1.97 & 0.23 & 5.2 & 0.81 \\
\hline 8 & 3.74 & 0.02 & 1.53 & 1.77 & 0.23 & 5.2 & 0.81 \\
\hline
\end{tabular}

\section{REFERENCES}

[1] L. Tang and B.-T. Ooi, "Locating and isolating DC faults in multiterminal DC systems," IEEE Trans. Power Del., vol. 22, no. 3, pp. 18771884, July 2007.

[2] — , "Protection of VSC-multi-terminal HVDC against DC faults," in Proc. IEEE 33rd Annual Power Electronics Specialists Conference pesc 02, vol. 2, Jun. 23-27, 2002, pp. 719-724.

[3] H. Chen, C. Wang, F. Zhang, and W. Pan, "Control strategy research of VSC based multiterminal HVDC system," in Proc. IEEE PES Power Systems Conference and Exposition PSCE 'O6, Oct. 2006, pp. 19861990.

[4] L. Tang and B.-T. Ooi, "Elimination of "harmonic transfer through converters" in VSC-based multiterminal DC systems by AC/DC decoupling," IEEE Trans. Power Del., vol. 23, no. 1, pp. 402-409, January 2008.

[5] F. Lin, Z. Ma, X. You, and T. Zheng, "The grid connected converter control of multi-terminal DC system for wind farms," in Proc. Eighth International Conference on Electrical Machines and Systems ICEMS 2005, vol. 2, Sep. 29-29, 2005, pp. 1021-1023.

[6] S. Zhou, "Control of multi-terminal VSC-HVDC transmission system," in Proceedings of the 44th International Universities Power Engineering Conference, University of Strathclyde, September 2009.

[7] C. Zheng, X. Zhou, and R. Li, "Dynamic modeling and transient simulation for VSC based HVDC in multi-machine system," in Proc. International Conference on Power System Technology PowerCon 2006, Oct. 22-26, 2006, pp. 1-7.

[8] S. Cole and R. Belmans, "Modelling of VSC HVDC using coupled current injectors," in Proc. IEEE Power and Energy Society General Meeting - Conversion and Delivery of Electrical Energy in the 21st Century, Jul. 20-24, 2008, pp. 1-8.

[9] K. R. Padiyar and N. Prabhu, "Modelling, control design and analysis of VSC based HVDC transmission systems," in Proc. International Conference on Power System Technology PowerCon 2004, vol. 1, Nov. 21-24, 2004, pp. 774-779.

[10] O. A. Giddani, G. P. Adam, O. Anaya-Lara, and K. L. Lo, "Grid integration of a large offshore wind farm using VSC-HVDC transmission system in parallel with AC submarine cable," in Proceedings of the 44th International Universities Power Engineering Conference, University of Strathclyde, September 2009.

[11] S. Lefebvre, W. Wong, J. Reeve, J.-M. Gagnon, and B. Johnson, "Experience with modeling MTDC systems in transient stability programs," IEEE Trans. Power Del., vol. 6, no. 1, pp. 405-413, January 1991.

[12] S. Lefebvre, W. Wong, J. Reeve, M. Baker, and D. Chapman, "Considerations for modeling MTDC systems in transient stability programs," IEEE Trans. Power Del., vol. 6, no. 1, pp. 397-404, January 1991.

[13] M. Kazmierkowski and L. Malesani, "Current control techniques for three-phase voltage-source PWM converters: a survey," IEEE Transactions on Industrial Electronics, vol. 45, no. 5, pp. 691-703, Oct. 1998.

[14] G. Terörde, Electrical Drives and Control Techniques. Acco, 2004.

[15] L. Zhang and H.-P. Nee, "Multivariable feedback design of VSC-HVDC connected weak AC systems," in PowerTech 2009, 2009, p. 8.

[16] C. Du, "VSC-HVDC for industrial power systems," Ph.D. dissertation, Chalmers University of technology, 2007.

[17] SimPowerSystems 5, User's Guide, Hydro-Québec. [Online]. Available: http://www.mathworks.com/access/helpdesk/help/pdf_doc/ physmod/powersys/powersys.pdf

[18] MATPOWER website. [Online]. Available: http://www.pserc.cornell. edu/matpower/

[19] MatDyn website. [Online]. Available: http://www.esat.kuleuven.be/ electa/teaching/matdyn/

[20] S. Cole and R. Belmans, "MatDyn, a new Matlab based toolbox for power stability studies, geared towards education and research," Submitted to IEEE Trans. on Power Systems, 2009. 


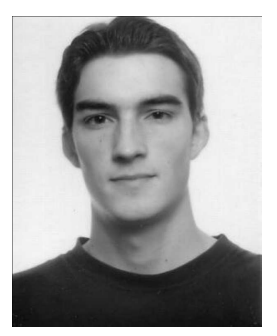

Stijn Cole (S'05) received the M.S. degree in electrical engineering in 2005 from the Katholieke Universiteit Leuven (K.U.Leuven), Belgium. Since 2005 he is working as a research assistant at K.U.Leuven. $\mathrm{He}$ is a member of ELECTA, the Electrical Energy research group, of the department of Electrical Engineering of the K.U.Leuven, where he is working towards a Ph.D. His fields of interest include power systems, grid of the future, and HVDC.

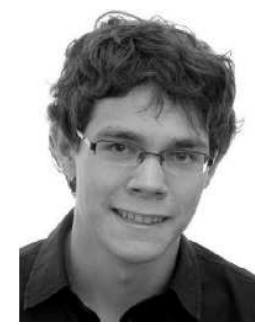

Jef Beerten (S'07) was born in Belgium in 1985. He received the M.Sc. degree in electrical engineering from the Katholieke Universiteit Leuven (K.U.Leuven), Leuven, Belgium, in 2008, where he is currently working towards the Ph.D. degree. He is a Research Assistant with the division ESATELECTA division of K.U.Leuven. His research interests include power system control, integration of renewables and multiterminal HVDC in particular. Mr. Beerten holds a Ph.D. fellowship from the Research Foundation Flanders (FWO).

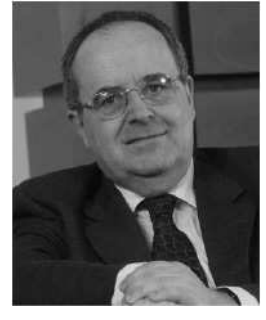

Ronnie Belmans (S'77-M'84-SM'89-F'05) received the M.S. degree in electrical engineering in 1979 and the Ph.D. degree in 1984, both from the K.U.Leuven, Belgium, the Special Doctorate in 1989 and the Habilitierung in 1993, both from the RWTH, Aachen, Germany. Currently, he is a full professor with the K.U.Leuven, teaching electric power and energy systems. His research interests include techno-economic aspects of power systems, power quality and distributed generation. He is also guest professor at Imperial College of Science, Medicine and Technology, London-UK. Since June 2002 he is chairman of the board of directors of ELIA, the Belgian transmission grid operator. 